\title{
OJ 287 - A SYSTEM WITH A BINARY BLACK HOLE
}

\section{HARRY J. LEHTO}

Tuorla Observatory, Turku University

Väisäläntie 20, FI-21500 Piikkiö, FINLAND

\section{Introduction}

OJ 287 is a blazar type active galaxy. The historical light curve shows outbursts occurring at quasi-regular intervals. These can be modeled by a secondary black hole interacting with the accretion disk of the primary black hole. We present results of wavelet analysis of the recent light curve of OJ 287, including the outbursts in 1994 and 1995.

We adopted a Morlet wavelet for this investigation. We have characterized the level of variability as a function of time and temporal frequency in the irregularly sampled light curve of OJ 287.

\section{Analysis}

Wavelets can decompose variability locally (around $t=\tau$ ) into different timescales or frequencies $(f)$. We have used a Morlet wavelet

$$
g^{*}(f, \tau)=\exp \left(-i c f(t-\tau)-\frac{1}{2}(f(t-\tau))^{2}\right), \text { where, } c=2 \pi,
$$

and calculated the transform $W(f, t)=f \cdot\left(S^{2}(f, \tau)+C^{2}(f, \tau)\right)$, where

$$
\begin{aligned}
& S(f, \tau)=\sum_{\text {all } j} m_{j} \sin \left(2 \pi f\left(t_{j}-\tau\right)\right) \exp \left(-\frac{1}{2} f^{2}\left(t_{j}-\tau\right)^{2}\right) \\
& C(f, \tau)=\sum_{\text {all } j} m_{j} \cos \left(2 \pi f\left(t_{j}-\tau\right)\right) \exp \left(-\frac{1}{2} f^{2}\left(t_{j}-\tau\right)^{2}\right) .
\end{aligned}
$$

In EVENLY SPACED DATA one may select $f_{i+1}=2 f_{i}$ and $\tau_{k+1}=\tau_{k}+$ $1 / f_{i}$. 
In UNEVENLY SPACED DATA sampling causes problems (Lehto, 1996) which can be avoided mostly by the next scheme:

(1) Bin locally the data on a grid much finer than $\delta t=1 / f$.

(2) Average values of each bin.

(3) For empty bins extrapolate the light curve from the binned values.

(4) Calculate the wavelet transform as in evenly spaced case.

Discreteness will cause undulations at low frequencies at the ends of data.

A strong sinusoid $A \sin (2 \pi t \cdot f), t_{o}<t<t_{1}$ transforms to $W=(\pi / 2)$. $\left(A^{2} / f\right)$, for timescales $t_{o}<t<t_{1}$.

\section{Variability}

Most of the large amplitudes features detected can be explained by the binary black hole model (LV96, VL97, Sundelius et al. 97).

1) The slowest variability is part of a long "baseline" cycle in OJ 287. The data is too short to give a correct estimate of the exact time scale.

2) The two major outbursts correspond to the ones described in LV96 and VL97. They are caused by an 100 million solar mass black hole plunging through the accretion disk of a $17 \cdot 10^{9} \mathrm{M}$. black hole.

3) The third outburst can be attributed to matter drawn from the accretion disk of the primary black hole by tidal interaction and radiating as it falls into the primary black hole (Sundelius et al. 97).

4) Short lived oscillations with periods of about 15-30 days are also apparent in the data. The period of the last stable orbit of the $1.7 \cdot 10^{10} M_{\odot}$ black hole is 118 days. The keplerian "orbital timescale" at the "surface" of the black hole is 23 days. The observed timescale could be caused by matter in its final fall into the black hole.

5) Seven episodes of very fast and short lived variations are seen. These timescales, clustering around $\tau=5$ days, can be explained by matter radiating in a helical jet with a helical cycle of about 23 days and Dopr boosted by $(\Gamma \sim 5)$, similar to observed VLBI boosting factors (Gabuzd: et al. 1989). Alternatively, they may be caused by material close to lasi stable orbits $(P=0.7 \mathrm{~d})$ around the $10^{8} \mathrm{M}_{\odot}$ secondary black hole.

Acknowledgements:

The data for this analysis was provided by the $0 \mathrm{~J}-94$ collaboration. References

Gabuzda, D.C., Wardle, J.F.C. and Roberts, D.H., 1989, Ap.J., 336, L59.

Lehto, H.J. 1996, "Wavelets in Unevenly Spaced Data: OJ 287 light curve" in Statistical Challenges in Modern Astronomy II, eds. G.J. Babu and E.D. Feigelson, p. 423.

Lehto H.J. and Valtonen M.J. 1996, Ap.J., 460, 207. (LV96)

Sundelius, B., Wahde, M., Lehto H.J. and Valtonen, M.J., 1997, Ap.J., 484, 180.

Valtonen M.J. and Lehto, H.J., 1997, Ap.J., 460, 207. (VL97) 